\title{
Psychoacoustic Properties of Fibonacci Sequences
}

\author{
J. Sokoll , S. Fingerhuth
}

1202, Fibonacci set up one of the most interesting sequences in number theory. This sequence can be represented by so-called Fibonacci Numbers, and by a binary sequence of zeros and ones. If such a binary Fibonacci Sequence is played back as an audio file, a very dissonant sound results. This is caused by the "almost-periodic", "self-similar" property of the binary sequence. The ratio of zeros and ones converges to the golden ratio, as do the primary and secondary spectral components intheir frequencies and amplitudes. These Fibonacci Sequences will be characterized using listening tests and psychoacoustic analyses.

Keywords: Fibonacci Sequence, psychoacoustics, listening tests.

\section{Introduction}

In 1202, the Italian mathematician Fibonacci (also known as Leonardo da Pisa) asked a simple question in his book Liber Abaci: If a pair of rabbits beget a pair of new rabbits after one year of maturing, and these rabbits beget another pair after maturing, how many pairs of rabbits will there be after $n$ years? To make things simple, Fibonacci assumed that rabbits never die and breed every year. Fig. 1 shows the first 5 years of the rabbit population.

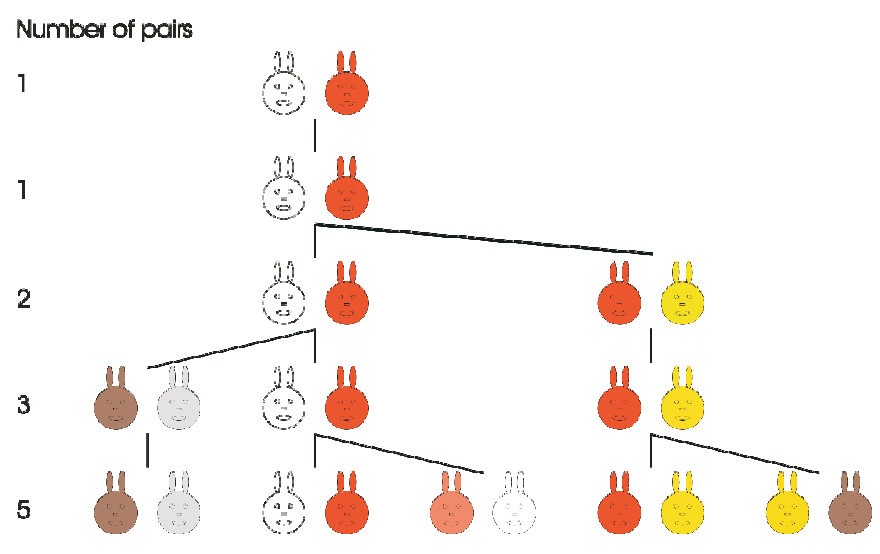

Fig. 1: The Fibonacci rabbit population

The number of rabbit pairs in the $n$th year $F_{n}$ ( $F$ for Fibonacci) is the number of pairs one year earlier $F_{n-1}$ (because the rabbits do not die) plus the number of pairs two years earlier $F_{n-2}$, because they are all mature and can reproduce:

$$
F_{n}=F_{n-1}+F_{n-2}
$$

Starting with one immature pair of rabbits $\left(F_{1}=1, F_{2}=1\right)$, it is easy to calculate the number of pairs for the next years:

$$
1,1,2,3,5,8,13,21,34,55,89,114,223,347 \ldots
$$

If an immature pair of rabbits is represented by a " 0 " and a mature pair is represented by a " 1 ", a binary Fibonacci Sequence can be built. In the first year a "0" would represent one immature pair, and in the second year a " 1 " would represent the matured pair. In the third year a "10" would represent the same matured pair and a newly born immature pair. Table 1 shows the binary Fibonacci Sequence for the first 8 years.

Table 1: The binary Fibonacci Sequence

\begin{tabular}{|c|c|c|c|c|c|}
\hline Year & & $\boldsymbol{F}_{\boldsymbol{n}}$ & $\boldsymbol{M}$ & $\boldsymbol{N}$ & $\boldsymbol{M} / \boldsymbol{N}$ \\
\hline 1 & 0 & 1 & 0 & 1 & 0 \\
\hline 2 & 1 & 1 & 1 & 0 & $\infty$ \\
\hline 3 & 10 & 2 & 1 & 1 & 1 \\
\hline 4 & 101 & 3 & 2 & 1 & 2 \\
\hline 5 & 10110 & 5 & 3 & 2 & 1.5 \\
\hline 6 & 10110101 & 8 & 5 & 3 & $1 . \overline{66}$ \\
\hline 7 & 1011010110110 & 13 & 8 & 5 & 1.6 \\
\hline 8 & 101101011011010110101 & 21 & 13 & 8 & 1.625 \\
\hline
\end{tabular}

The self-similarity of the Fibonacci Sequence can also be found in the binary sequence: After the first two years, the $n$th binary sequence can be built if the sequence of year $n-2$ is attached to the end of the sequence of year $n-1$.

The sequence of the number of pairs $F_{n}$ is the original Fibonacci Sequence. The number of mature pairs ("1") $M$ are the Fibonacci Numbers starting in year 2, and the number of immature pairs (" 0 ") $N$ are the Fibonacci Numbers starting in year 3 . The quotient of the ones and zeros $M / N$ converges to the Golden Ratio:

$$
\frac{M}{N}=\frac{F_{n}}{F_{n-1}} \stackrel{n \rightarrow \infty}{\longrightarrow} g .
$$

The Golden Ratio is a geometric relation: A straight line is sectioned in such a way that the ratio of the total length to the longer segment equals the ratio of the longer segment to the shorter one. Calling the total length $l$ and the longer segment $a$, the following equation describes the Golden Ratio:

$$
g:=\frac{l}{a}=\frac{a}{l-a} .
$$

The only positive solution of this equation is 


$$
g=\frac{1+\sqrt{5}}{2}=1.618033989 \ldots
$$

The Golden Ratio is considered the most irrational of all ratios. The Fibonacci Number $F_{n}$ can be easily calculated with this approximation (the wavy equal sign means: take the nearest integer) [1]:

$$
F_{n} \approx \frac{g^{n}}{\sqrt{5}}
$$

\section{Acoustical realization of the Fibonacci Sequence}

As a very dissonant sound is expected if the binary Fibonacci Sequence is played as an audio file, it would be interesting to analyze these sounds. For the acoustical realization of the Fibonacci Sequence, a binary sequence of any length $F_{n}$ can be generated and played back with a digital-analog-converter. Although there is no definite period, certain sequences recur and the signal shows a distinct discrete spectrum. The analysis of longer sequences shows only small differences, so that the spectrum of a sequence with a length of $F_{20}=6765$, as shown in Fig. 2 can be seen as the spectrum of a sequence of infinite length [2].

In the spectrum of the Fibonacci Sound, the Golden Ratio $g$ can be found again. In the top plot of Fig. 2 the second highest peak has the magnitude of the highest peak divided by $g$ $(1 / g=0.618)$ and the frequency of the highest peak multiplied by $g(392 \cdot g=634)$.

The binary Fibonacci Sequence was interpolated with a sinc-algorithm to minimize the spectral influences of the rectangle-characteristic of a binary sequence. Because of the low-pass characteristic of the sinc-interpolation, the high spectral components were cut off. The sinc-interpolated binary Fibonacci Sequences were integrated in an internally developed wavetable synthesizer (see Fig. 3). With this synthesizer it is possible to play intervals and melodies with Fibonacci Sounds. MIDI files are supported. To characterize the psychoacoustic properties of the Fibonacci Sequences, listening tests and psychoacoustic analyses have been performed. For the listening tests, sequences of length $F_{16}=987$ have been used.

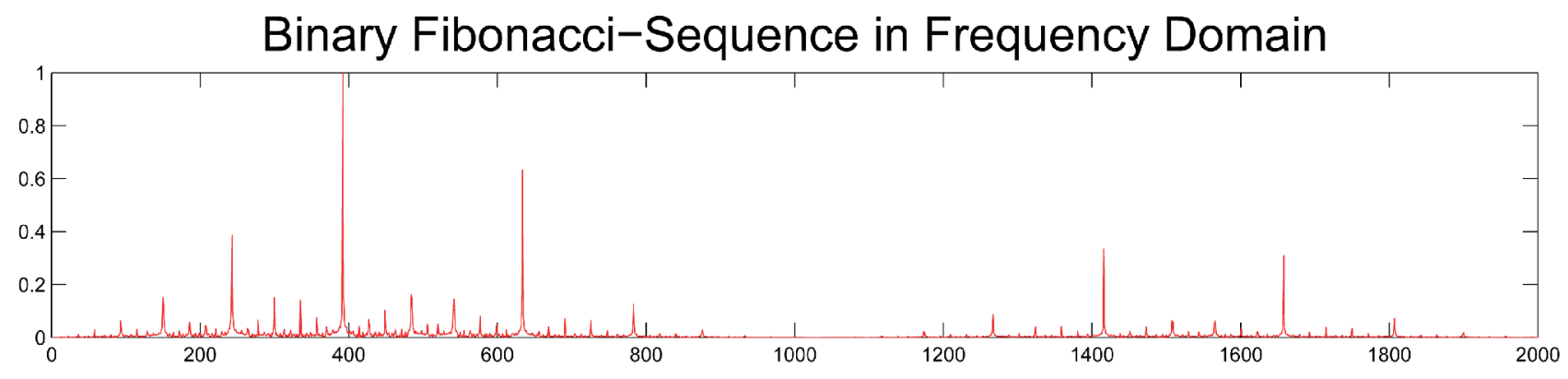

\section{Sinc Interpolation of Binary Fibonacci-Sequence in Frequency Domain}

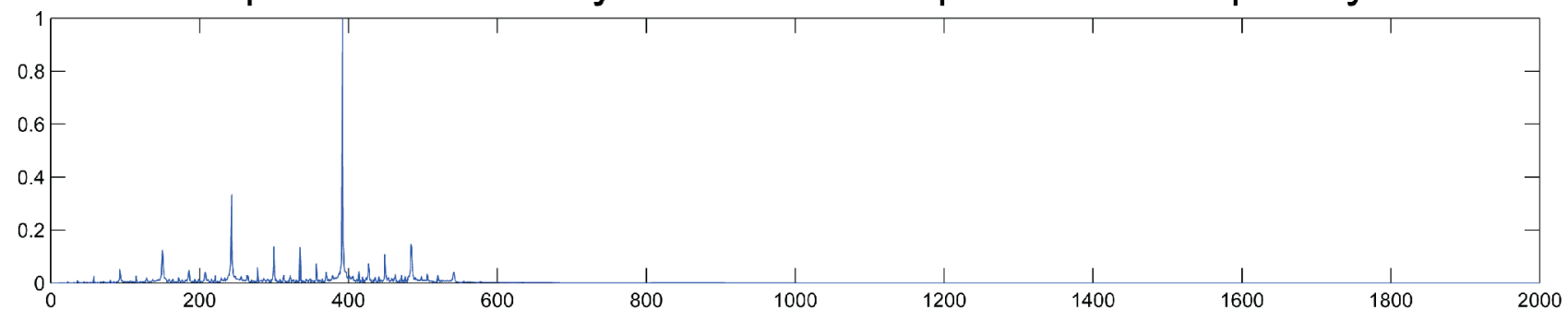

\section{Binary Fibonacci-Sequence and Sinc Interpolation of Binary Fibonacci-Sequence in Time Domain}

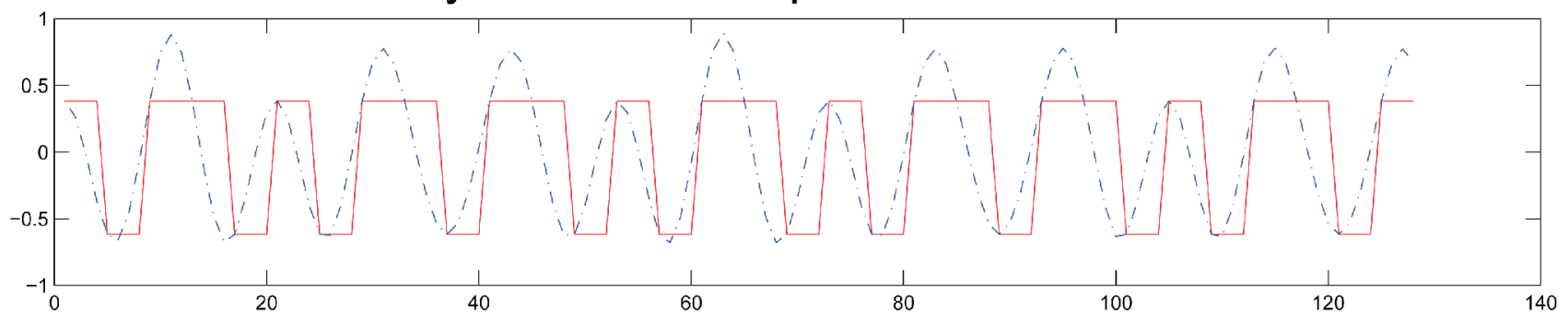

Fig. 2: Binary sequence vs sinc interpolation 


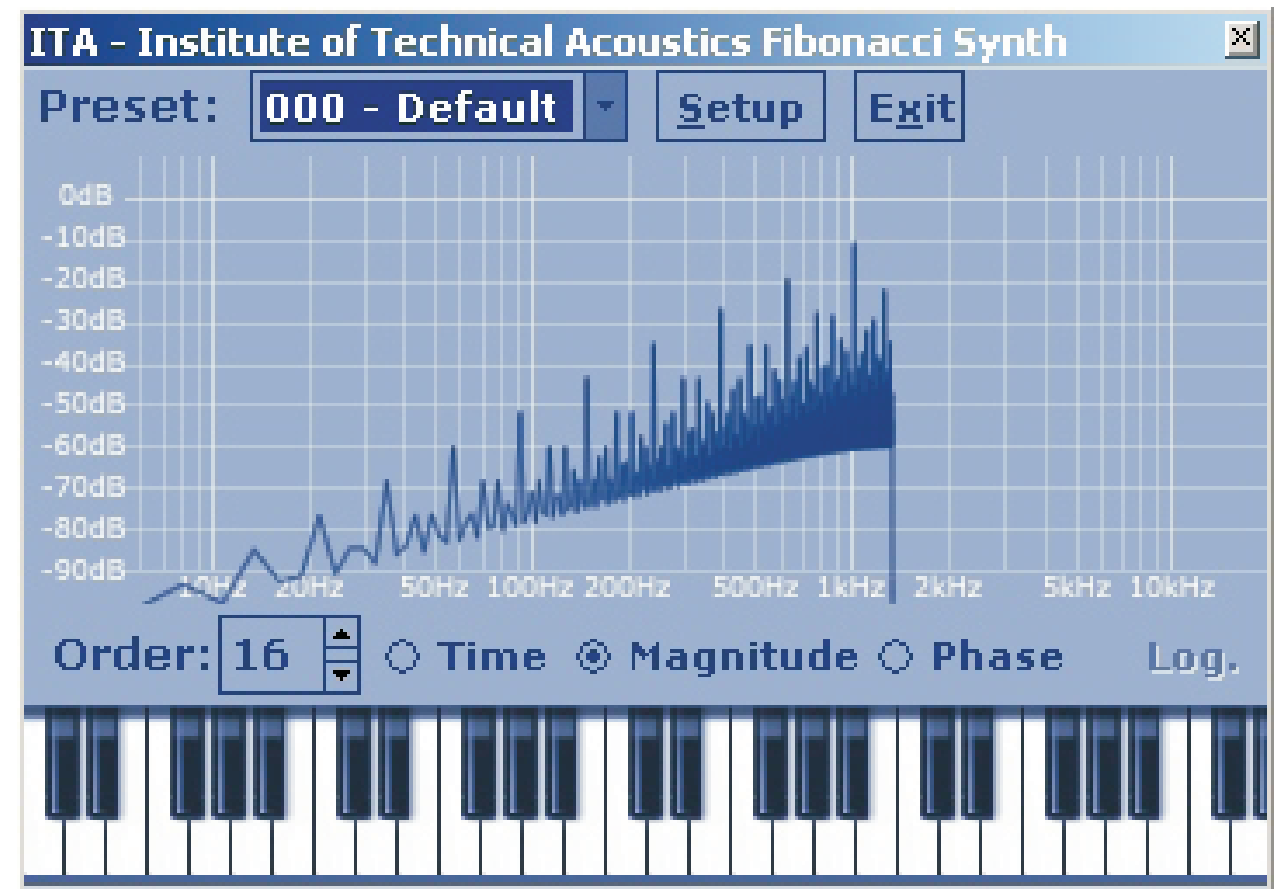

Fig. 3: The Fibonacci Synthesizer

\section{Listening tests}

For a subjective psychoacoustic analysis, listening tests have to be performed. In the first test the subjects heard popular melodies played with the Fibonacci Synthesizer. All subjects were able to recognize the melodies, though the subjects could not identify the intervals. A "Fibonacci octave" cannot be perceived as a "real" octave with a frequency ratio of 1:2. The Tritone Paradox phenomenon [4] occurs as well.

[start frequency: result of last pass] [start frequency: $0 \mathrm{~Hz}$ ]

[start frequency: $10 \mathrm{kHz}$ ]
The next test examined whether one of the peaks in the spectrum could be spotted as the most remarkable peak. The subjects were told to change the pitch of a sine tone until it matched the Fibonacci Sound. The sounds were presented with an electrostatic Stax headphone with a linear frequency response: the pure tone on one ear, the Fibonacci Sound on the other ear. The effect of the binaural beat [3] was intentionally used to help to find the right pitch. As there is more than one noticeable peak in the spectrum of the Fibonacci Sounds, different subjects identified different peaks. Fig. 4 shows the results of this listening test. Each dot represents the choice of one subject.

\section{G3}

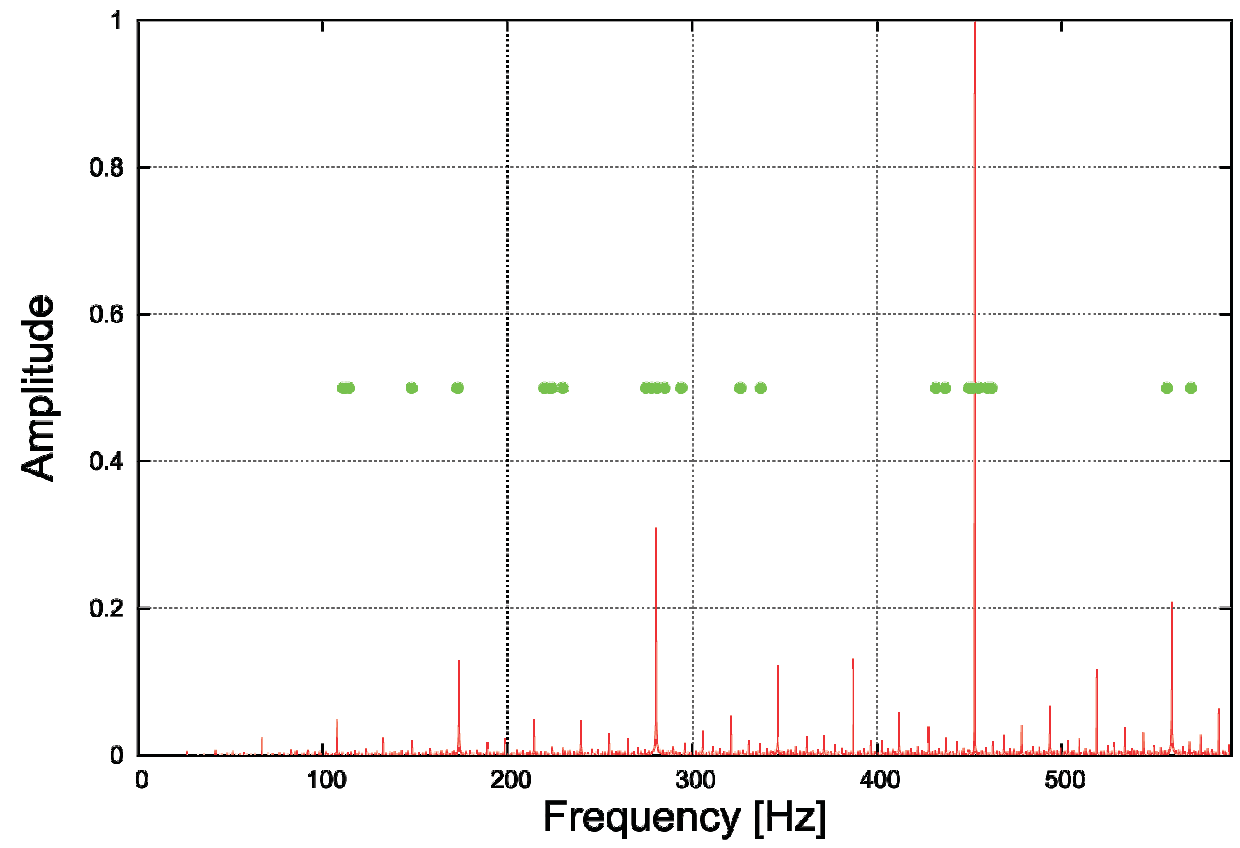



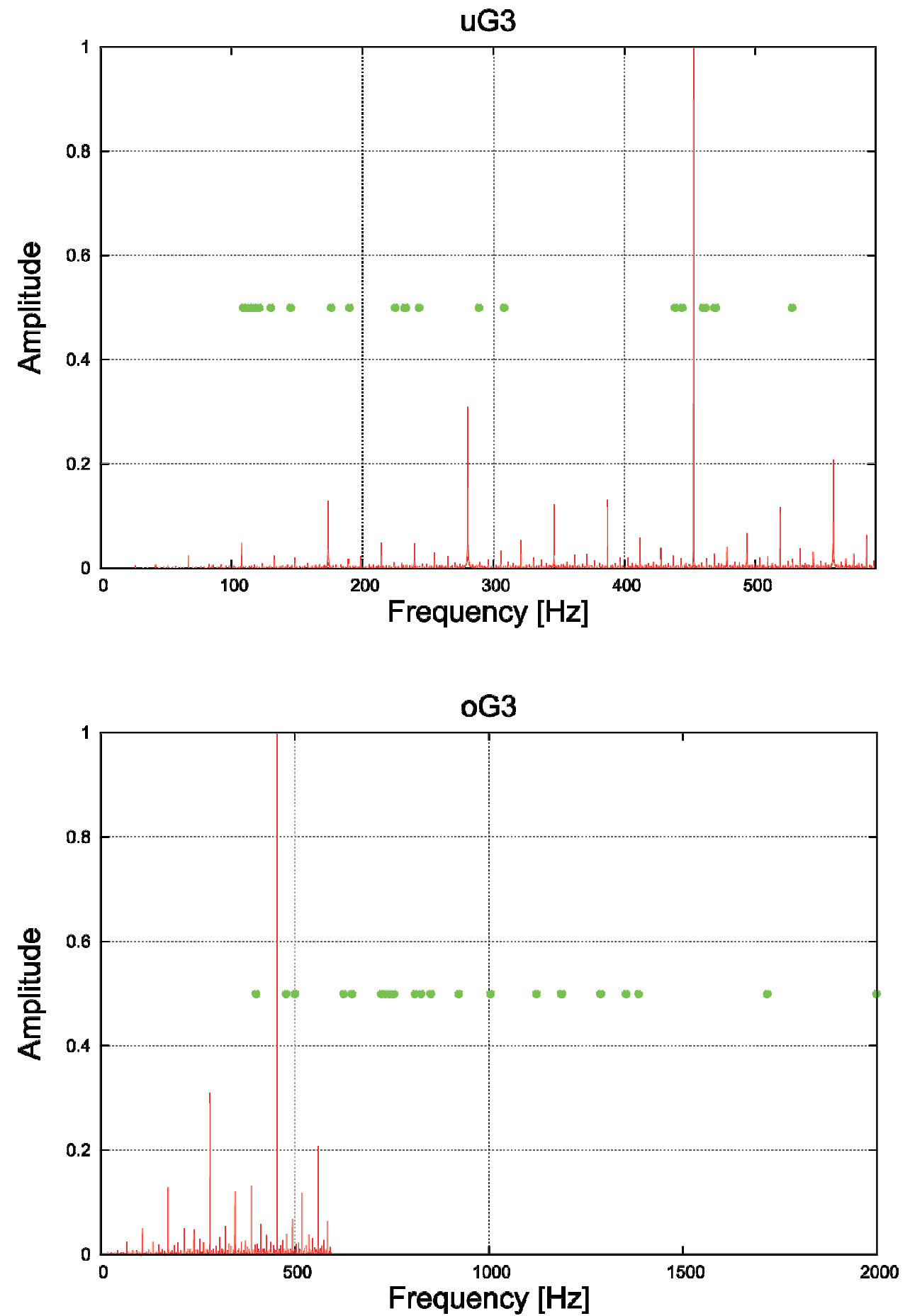

Fig. 4: Listening test: pitch-perception of Fibonacci Sounds

Because the subjects started each pass with the sine frequency of the last pass, two further tests were performed to find out whether the start frequency of the sine tone has an influence on the results. The subjects were asked to start each pass with the lowest sine tone of the frequency synthesizer and the highest respectively. Fig. 4 shows the results of the listening test starting each pass with a sine frequency of $0 \mathrm{~Hz}$. It can be seen that many more low frequencies were identified than in the first test. In the last listening test every pass started with a sine frequency of $10 \mathrm{kHz}$ (see Fig. 4). Almost all subjects have marked frequencies above the actual spectrum of the presented sounds.

The different results of the three different listening tests refer to the dissonant spectrum of the Fibonacci Sounds. Although there is a major peak in the frequency domain which is higher in amplitude by factor $g$ than the second major peak, this peak was only rarely identified in the pitch perception test. 


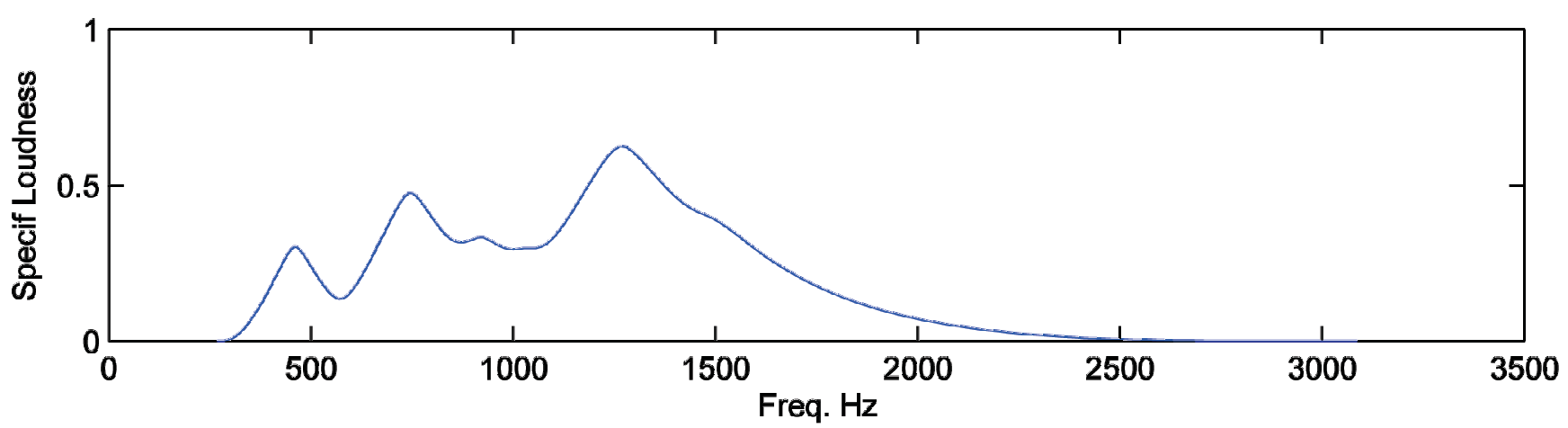

Fig. 5: Loudness of a Fibonacci Sound

\section{Psychoacoustics}

For an objective analysis, psychoacoustic parameters like loudness and Pitch Strength were calculated. Fig. 5 shows the loudness according to ISO 226:2003 [5] of a Fibonacci Sound.

The relative Pitch Strength [6] was calculated for the Fibonacci Sequence that was used in the listening tests. If a sine of $80 \mathrm{~dB}$ and $1500 \mathrm{~Hz}$ has a Pitch Strength of 1, the Fibonacci Sequence has a relative Pitch Strength of 0,1878 .

\section{Summary / Outlook}

The Fibonacci Numbers are a very interesting mathematical phenomenon. As the Golden Ratio $g$ occurs permanently in the spectrum of the binary Fibonacci Sequence, a very dissonant sound results. Although melodies can be recognized, it is not possible to identify specific intervals. Listening tests were performed in order to find the subjective pitch of the signals. It is not possible to define one most outstanding peak, as all subjects marked different frequencies.

In the future, more psychoacoustic analyses will be performed. Another project of the Institute of Technical Acoustics will investigate consonance and tonality.

\section{Acknowledgements}

The authors would like to thank Prof. Michael Vorländer for supporting this work and discussion, and also all the participants in the listening tests.

\section{References}

[1] Schroeder, M. R.: Number Theory in Science and Communication. $3^{\text {rd }}$ Edition. Springer Verlag 1999.

[2] Schmidt, H.: Eigenschaften und Akustische Realisierung von Fibonacci-Folgen. Proceedings of DAGA '88, Braunschweig, p. 621.

[3] Terhardt, E.: Akustische Kommunikation. Springer Verlag 1998.

[4] Deutsch, D.: The Tritone Paradox: Effects of Spectral Variables. Perception \& Psychophysics, Vol. 41 (1987), p. 563-575 .

[5] ISO 226:2003: Acoustics - Normal Equal-Loudness-Level Contours.

[6] Fruhmann, M.: Introduction and Practical use of an Algorithm for the Calculation of Pitch Strength. Journal of the Acoustical Society of America (JASA), Vol. 118 (2005), No. 3, Pt. 2 , p. 1894.

Jan Sokoll

e-mail: jso@akustik.rwth-aachen.de

Sebastian Fingerhuth

sfi@akustik.rwth-aachen.de

Institute of Technical Acoustics

RWTH Aachen University

D-52056 Aachen, Germany 